\title{
MECP2 disorders: from the clinic to mice and back
}

\author{
Laura Marie Lombardi, ${ }^{1,2,3}$ Steven Andrew Baker, ${ }^{2,4,5}$ and Huda Yahya Zoghbi $\mathbf{i}^{1,2,3,4}$ \\ 'Department of Molecular and Human Cenetics, Baylor College of Medicine, Houston, Texas, USA. ${ }^{2}$ Jan and Dan Duncan Neurological Research Institute at Texas Children's Hospital, Houston, Texas, USA. \\ ${ }^{3}$ Howard Hughes Medical Institute, ${ }^{4}$ Program in Developmental Biology, and ${ }^{5}$ Medical Scientist Training Program, Baylor College of Medicine, Houston, Texas, USA.
}

\begin{abstract}
Two severe, progressive neurological disorders characterized by intellectual disability, autism, and developmental regression, Rett syndrome and MECP2 duplication syndrome, result from loss and gain of function, respectively, of the same critical gene, methyl-CpG-binding protein 2 (MECP2). Neurons acutely require the appropriate dose of MECP2 to function properly but do not die in its absence or overexpression. Instead, neuronal dysfunction can be reversed in a Rett syndrome mouse model if MeCP2 function is restored. Thus, MECP2 disorders provide a unique window into the delicate balance of neuronal health, the power of mouse models, and the importance of chromatin regulation in mature neurons. In this Review, we will discuss the clinical profiles of MECP2 disorders, the knowledge acquired from mouse models of the syndromes, and how that knowledge is informing current and future clinical studies.
\end{abstract}

\section{Introduction}

Austrian pediatrician Andreas Rett first reported in 1966 on young females exhibiting progressive dementia, motor loss, and stereotyped hand movements following normal development in their first 1 to 1.5 years of life (1). He filmed their unique clinical presentation, attempting to raise awareness and find similar cases of what he termed "cerebral atrophy with hyperammonemia." Unfortunately, increased awareness of the condition occurred slowly due to the relative rarity of the condition, the fact that Dr. Rett published almost exclusively in German, and an equipment error resulting in only apparent hyperammonemia. It was not until 1983, when Swedish neurologist Bengt Hagberg and colleagues synthesized a series of similar cases from Europe in the Annals of Neurology, that the striking regression disorder would truly enter the international medical consciousness (2). In honor of the original observer and the continued dedication of Dr. Andreas Rett to these patients, the disorder would henceforth be known as Rett syndrome.

In the years that followed, a clear clinical picture began to crystallize. Rett syndrome occurs in 1 in 10,000 live female births and is sporadic in over $99 \%$ of the cases (3). What genetic or environmental insult could result in such an acute defect around the peak of infant synaptogenesis? In 1999, Dr. Ruthie Amir, a postdoctoral fellow, discovered that loss-of-function mutations in methylCpG-binding protein 2 (MECP2) formed the genetic basis of Rett syndrome (4). Less than 6 years later, duplication of the very same gene was implicated in a severe male intellectual disability syndrome associated with premature death, MECP2 duplication syndrome (MDS) (5). In this Review, we will discuss the clinical consequences of MECP2 loss and gain of function, the power and significance of mouse models of these disorders, our molecular knowledge of MeCP2, and the path these models and molecular knowledge have paved for clinical trials.

Conflict of interest: Huda Yahya Zoghbi is a co-holder of U.S. Patent 6,709,817, "Method of Screening Rett Syndrome by Detecting a Mutation in MECP2," March 23, 2004.

Reference information: J Clin Invest. 2015;125(8):2914-2923. doi:10.1172/JCI78167.

\section{Manifestations of disease}

MECP2 dosage sensitivity. In their landmark 1983 article, Hagberg and colleagues catalogued 35 patients encountered over the course of 21 years in three different European countries (2). This breadth of clinical experience enabled them to propose four distinct phases of Rett syndrome: (a) stagnation of development after 7 to 18 months, (b) rapid deterioration, (c) a pseudostationary phase, and (d) late motor deterioration (6). Developmental stagnation is typified by a failure to meet major developmental milestones, including word development, social interaction, and motor ability. This developmental stagnation is further evidenced by acquired microcephaly and overall growth delay. Rapid deterioration results in loss of previously acquired abilities, such as walking or pulling to a stand, word use or babbling, affinity for or attention to social interaction, and grasping or gesturing. Purposeful hand movements are replaced by stereotypies, such as hand clasping and wringing. Respiratory abnormalities, such as hyperventilation and apneas, also often emerge at this stage. Mental decline coincides with motor dysfunction characterized by apraxia and often an ataxic gait. In the so-called pseudostationary phase (onset at $\sim 3$ to 10 years of age), seizures and scoliosis are common, but social interaction defects subside somewhat. Eventually, many patients lose the ability to ambulate and sometimes develop parkinsonian features in the phase of late motor deterioration $(7,8)$. These clinical features occur in the absence of any evidence of neuronal degeneration but are accompanied by decreased brain size, most likely resulting from the smaller neurons and reduced dendritic branching (9).

The apparent gender specificity of this disorder was just as puzzling as the clinical presentation of regression. To explain this "exclusive involvement of females," Hagberg proposed early on that Rett syndrome was due to a dominant X-linked mutation that resulted in nonviable hemizygous males (2). Further, he argued that his model predicted the rare occurrence of Klinefelter males (XXY) with Rett symptoms. Indeed, such a male was identified in 1999 (10). Although 99\% of Rett cases are sporadic, the genetic basis of the disorder was affirmed by multiple examples of identical twin sisters concordant for Rett syndrome $(11,12)$. In the 


\begin{tabular}{|c|c|c|c|c|c|}
\hline & \multirow[b]{2}{*}{ Rett } & \multicolumn{2}{|c|}{ Null mice } & \multirow{2}{*}{$\begin{array}{c}\text { Mecp2R308X } \\
\text { Males }\end{array}$} & \multirow[t]{2}{*}{ Refs. } \\
\hline & & Males & Het females & & \\
\hline \multirow[t]{5}{*}{ Motor } & Limited mobility & + & + & + & $44,141-143$ \\
\hline & Ataxic gait & + & + & + & $43,44,144$ \\
\hline & Dystonia/rigidity & + & + & + & 44,143 \\
\hline & Tremor & + & + & + & 43,143 \\
\hline & Stereotypies & - & - & + & 143 \\
\hline \multirow[t]{4}{*}{ Cognitive and social abilities } & Decreased cognition & + & + & + & $53,141,144$ \\
\hline & Speech loss & ND & ND & ND & \\
\hline & Social avoidance & - & + & - & 142 \\
\hline & Anxiety & * & * & + & $141-144$ \\
\hline \multirow[t]{2}{*}{ Morphological } & Microcephaly & + & - & - & $43,121,144$ \\
\hline & Neuronal hypotrophy & + & + & - & $43,121,145$ \\
\hline \multirow[t]{2}{*}{ Autonomic dysfunction } & Breathing abnormalities & + & + & ND & $132,142,146,147$ \\
\hline & Reduced lifespan & + & + & + & $44,121,143,148$ \\
\hline Other & Seizures & + & - & + & 143,149 \\
\hline
\end{tabular}

+, observed in multiple studies; *, decreased; -, not present; ND, not determined. Het, heterozygous; Refs., references.
Table 1. Face validity of MeCP2 loss-of-function mouse models

ability (5). After the incorporation of many more cases, it has been determined that the common minimal duplicated region includes only $M E C P 2$ and the adjacent gene IRAK1 (34-36). Males with MDS exhibit infantile hypotonia, autistic features, gait abnormalities, seizures, and recurrent infections (5, 36-39). The inclusion of IRAK1, which encodes IL receptor-associated kinase 1 , in the duplicated region was hypothesized to mediate the immunological defects of the patients; however, recent work indicates that overexpression of MECP2 is sufficient to impair $\mathrm{T}$ cell function (40). Though approximately handful of familial examples, skewed $\mathrm{X}$ chromosome inactivation (XCI) was observed in asymptomatic mothers but not in the affected daughters, suggesting that carrier mothers preferentially inactivated the mutant allele in the majority of cells (13-15). Determining the causative gene on the $\mathrm{X}$ chromosome relied on painstaking exclusion mapping of rare familial cases (16). Once the candidate region was narrowed down, sequencing of candidate genes in sporadic cases revealed mutations in MECP2 (4). Indeed, more than $95 \%$ of patients diagnosed with typical Rett syndrome have mutations in MECP2 (17).

Knowing the causative gene on the $\mathrm{X}$ chromosome clarified the striking female bias of the disorder. First, genotyping revealed that the majority of MECP2 mutations arise in the paternal germline $(3,18)$, helping explain the preponderance of sporadic female patients, since only females inherit a paternal $\mathrm{X}$ chromosome. Secondly, in the rare cases of familial transmission, mothers with favorably skewed XCI patterns can transmit a mutant allele to viable hemizygous male offspring, but these males typically die in infancy if they inherit total loss-of-function alleles $(13,19)$. Males with more mild mutations survive, exhibiting varying degrees of intellectual disability and, frequently, neuropsychiatric symptoms, such as bipolar disorder (20), schizophrenia (21), and attention deficit/hyperactivity disorder (ADHD) $(22,23)$. Importantly, these male patients helped broaden the phenotypic spectrum due to MECP2 mutations. We now know that females with psychiatric conditions can also have mild loss-of-function mutations in MECP2 $(22,24)$. Additionally, MECP2 mutations have been found in individuals diagnosed with autism (25-27), consistent with the finding of autistic features in girls with Rett syndrome (28-32).

Given the discovery that males with MECP2 mutations are born alive and suffer severe neurological phenotypes, Meins and colleagues evaluated the gene in a male with severe intellectual disability and discovered a duplication spanning the MECP2 locus (33). Shortly thereafter, duplications spanning MECP2 were observed in four familial cases of males with severe intellectual dis-
$40 \%$ of MDS males die before the age of 25 (41), again, females are more protected due to the presence of a WT X chromosome. Unlike Rett syndrome, this disorder is largely familial, as female carriers are healthy enough to transmit the allele. However, the health of female carriers appears to correspond to the extent of favorable XCI. Female carriers with $85 \%$ or more cells expressing the WT X chromosome exhibit anxiety and depression (38) but do not exhibit intellectual disability (5), whereas carriers with less favorable skewing exhibit mild intellectual disability and Rettlike phenotypes (42).

Mouse models of loss and gain. After establishing that loss-offunction mutations in MECP2 cause Rett syndrome, two Mecp2null mouse models were successfully generated in $2001(43,44)$. Female mice heterozygous for the null allele are initially normal but slowly develop a stiff, uncoordinated gait, breathing difficulties, and hindlimb clasping; they are hypoactive starting at about 16 weeks of age $(43,44)$. Hemizygous males exhibit a similar but more rapid regression, with approximately $50 \%$ dying by 8 to 11 weeks of age. Similar to the neuroanatomical findings in humans, murine Rett models also display microcephaly, without gross neuropathological changes or neurodegeneration. Specifically, mouse and human neurons without MeCP2 have smaller somas (43, 45, 46) and decreased dendritic complexity (47-51). A decrease in synaptic plasticity, or the ability of neurons to change their synaptic strength in response to activity, is also observed in many neuronal types (52-54). Abnormalities in neurotransmitter concentrations occur in KO mice (55-59) as well as in patients with Rett syndrome (60-62). Thus, the major human phenotypes of Rett syndrome are recapitulated in mice, allowing for functional disease dissection in this model system (Table 1 ).

Though Mecp2 is broadly expressed, animals with the null mutation only in the CNS were indistinguishable from those with the mutation throughout the body $(43,44)$. This strongly indicated that the exhibited movement and breathing defects stemmed from loss of Mecp2 specifically in the CNS. Further, the notion 
Table 2. Symptoms of the MDS mouse model

\begin{tabular}{|c|c|c|c|}
\hline & MDS symptom & TG1 males & Refs. \\
\hline \multirow[t]{4}{*}{ Motor } & Limited mobility & + & 72 \\
\hline & Ataxic gait & + & 72 \\
\hline & Stereotypies & + & 72 \\
\hline & Spasticity & + & 72 \\
\hline \multirow[t]{4}{*}{ Cognitive and social abilities } & Decreased cognition & * & 72 \\
\hline & Speech loss & ND & \\
\hline & Social avoidance & + & 73 \\
\hline & Anxiety & + & 73 \\
\hline \multirow[t]{4}{*}{ Other } & Recurrent infections & ND & \\
\hline & Reduced lifespan & + & 72 \\
\hline & Seizures & + & 72 \\
\hline & Dysmorphic features & ND & \\
\hline
\end{tabular}

+, observed in multiple studies; ${ }^{*}$, increased.

that $\mathrm{MeCP} 2$ is required for general neuronal health is underscored by the dysfunction associated with the many different neuronspecific Mecp2 KOs $(43,61,63-65)$. In contrast, removal of MeCP2 from astrocytes or microglia results in mild behavioral phenotypes without affecting lifespan $(66,67)$. Thus, the function of MeCP2 in the glial cell types assayed does not appear to drive pathogenesis, though Mecp2 expression in astrocytes improves the breathing symptoms of mice lacking MeCP2 in all other cell types (66). Consistent with a primarily neuron-driven pathogenesis, Mecp2 expression only in postmitotic neurons normalized brain weight and activity (68) and extended lifespan beyond 8 months of age, despite incomplete Mecp2 expression (70\%-90\% of neurons, depending on region) (69). Taken together, these data strongly suggest that loss of MeCP2 function in neurons is the dominant cause of dysfunction in Rett syndrome.

Given the severity of the Mecp2-null mutant models, two main questions emerged: (a) When is MeCP2 required, and (b) is this damage reversible, given that neurons lacking MeCP2 do not die? To determine whether there was a critical window in which neurons required $\mathrm{MeCP} 2$ or whether $\mathrm{MeCP} 2$ was required constantly, Mecp2 expression was halted in adult mice (aged 2 months). Interestingly, after deletion of Mecp 2 in the adult, half of the males die within 10 weeks, which is very similar to the timespan between birth and death seen in mice with germline deletion (70). Thus, $\mathrm{MeCP} 2$ is required throughout postmitotic neuronal life. To test the reversibility of Rett symptoms, Mecp2 expression was activated after the onset of neurological symptoms. This expression drastically decreased the severity of ataxia, hindlimb clasping, tremor, and irregular breathing and extended lifespan $(54,71)$. Therefore, a lack of MeCP2 does not result in irreversible neuronal damage, prompting hope for benefits from potential therapeutic treatments in patients with Rett syndrome.

Fortuitously, a mouse model generated in 2004 revealed that overexpression of MeCP2 also results in neuronal dysfunction, predicting human MDS (72). Specifically, mice that overexpress MeCP2 exhibit a progressive neurological disorder characterized by hypoactivity, social avoidance, anxiety, and seizures (Table 2 and refs. $68,72,73)$. Similar to the increased severity of patients with MECP2 triplication compared with those with MDS (74), the more MeCP2 expressed by mice, the worse their phenotype $(72,73)$. Strikingly, while neurons without MeCP2 exhibit a decrease in excitatory synapses and impaired long-term potentiation (LTP), neurons expressing twice the normal level of MeCP2 have an increased density of these synapses (75) and enhanced LTP (72). This apparent increase in excitability is correlated with the enhanced motor-learning ability of the mice (72). Thus, humans and mouse models of both loss and gain of MeCP2 function indicate that neurons are acutely sensitive to the dose of MeCP2, with too little or too much resulting in progressive neurological disorders.

\section{From patient to protein: the molecular function of MeCP2}

Methylation binding and beyond. Before mutations in MECP2 were known to form the genetic basis of Rett syndrome, MeCP2 had already been biochemically characterized as interacting with methylated cytosines, followed by a guanine via its methyl-CpGbinding domain (MBD) (Figure 1A and refs. 76, 77). Recently, it was discovered that the MBD of MeCP2 also binds methylated cytosines in the $\mathrm{CH}$ context (where $\mathrm{H}=\mathrm{A} / \mathrm{T} / \mathrm{C}$ ), with similar affinity both in vitro and in vivo (78-80). DNA methylation is a covalent modification of the genome that provides an additional layer of information beyond the sequence information; thus, it is considered an epigenetic mark. DNA methylation is critical to the processes of genomic imprinting, XCI, cellular differentiation, and the silencing of transposable elements $(81,82)$. Constitutive heterochromatin, such as pericentromeric repeats, is characterized by high levels of DNA methylation, is important for the structural integrity of the centromere, and is, as anticipated, highly enriched for MeCP2. However, DNA methylation is also distributed throughout the genome in intergenic regions, within enhancers, proximal to the transcription start site (TSS), and within gene bodies. The complexity of the modification, and hence a protein targeted by the modification, lies in its context dependency. For example, while methylation near the TSS is associated with long-term repression of a gene, methylation within gene bodies is not $(83,84)$. Furthermore, methylation in the adult brain is very unique among somatic tissues, given its high $\mathrm{mCH}$ content $(\sim 25 \%$ of $\mathrm{mC})(78,85)$. Strikingly, in both mice and humans, $\mathrm{mCH}$ emerges only as neurons mature (in 2- to 4-week-old mice), paralleling the increased abundance of $\operatorname{MeCP} 2(86,87)$ and the global increase in synapse formation (85). Though we are arguably in the early stages of understanding the functional impact of $\mathrm{mCH}-$ as its relationship to expression varies in different cell types (85) transcriptional changes observed in $\mathrm{KO}$ and $M E C P 2$-overexpressing animals correlate more with $\mathrm{mCH}$ than with $\operatorname{mCG}(79,80)$.

Given the presence of an MBD, early functional studies of $\mathrm{MeCP} 2$ operated under the assumption that MeCP2 was likely a transcriptional repressor. Indeed, in vitro transcriptional reporter assays using methylated templates indicated that MeCP2 was capable of repression via a region mapped to amino acids 207-310, named the transcriptional repression domain (TRD) (Figure 1A and ref. 88). However, in vivo transcriptional studies in many different brain regions comparing the $\mathrm{KO}$ transcriptome with that of mice overexpressing MECP2 indicated that the majority of misregulated genes are not upregulated in the $\mathrm{KO}$ mice, as is often observed upon mutation of a repressor $(80,89,90)$. Although careful stud- 
A Canonical MeCP2 domain structure

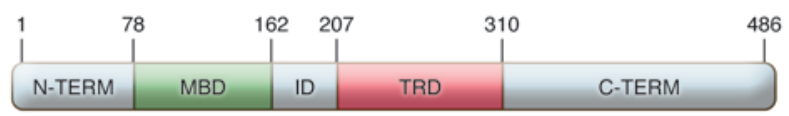

\section{B Conserved MeCP2 domains}

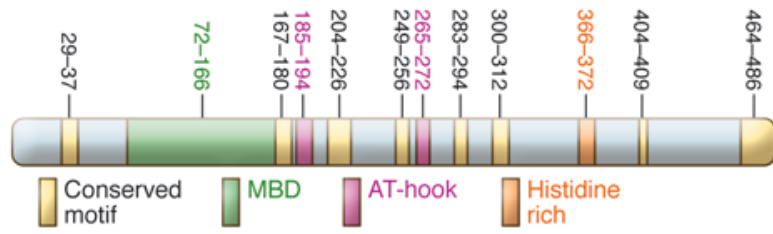

C Established Rett-causing mutations

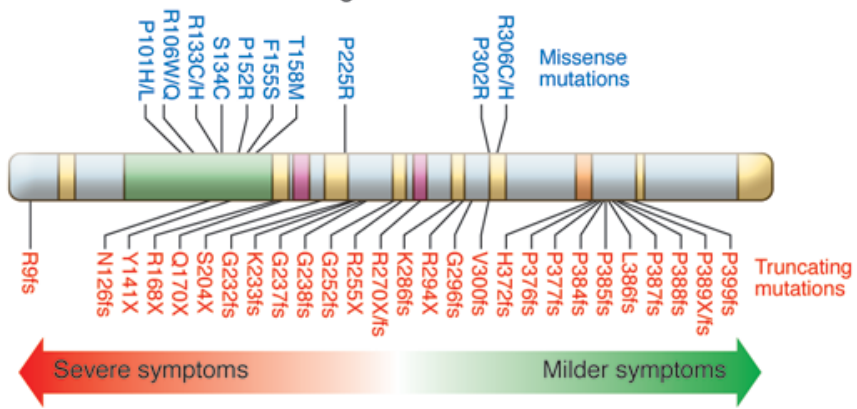

ies have not been performed to distinguish primary and secondary transcriptional effects, the fact that the majority of transcriptional changes are reciprocal between the KO and the MECP2-overexpressing mice suggests a nonrandom sensitivity of genes to MeCP2 function. Further, in contrast to derepression, a global decrease in transcription is observed in neurons derived from mouse and human embryonic stem cells lacking $\operatorname{MeCP} 2(46,91)$.

It is important to consider the biochemical properties of $\mathrm{MeCP} 2$ when investigating the mechanistic details of its function. Circular dichroism spectroscopy indicates that the full-length protein is approximately $59 \%$ unstructured in solution (92). Trypsin digestion of MeCP2 supports deletion-mapping studies used to define the MBD and TRD, as these functional domains can be recovered intact at high trypsin concentrations, suggesting that they are structurally discrete (92). However, only the structure of the MBD has been successfully solved $(93,94)$. The isoelectric point of the full-length protein is highly basic $(\sim 10)$, suggesting the capacity for multiple DNA interaction regions. Indeed, many portions of MeCP2, aside from the MBD, have been shown to interact with DNA in a methylation-independent manner, including the TRD and the interdomain (ID) between the MBD and TRD (95). In fact, in 1992, with the initial cloning of Mecp2, the authors were struck by the abundance of short basic motifs in the protein, which were known at the time to facilitate DNA binding (76). However, it was not until recently that comparative computational analysis facilitated the mapping of three AT-hooks in MeCP2, which are specialized basic clusters that bind the minor groove of AT-rich DNA (Figure 1B and refs. 96). Further, comparing two patient mutations with and without AT-hook 2, G273X and R270X, respectively, established that AT-hook 2 contributes to MeCP2 DNA binding in vivo and in vitro (96). Importantly, this difference in DNA binding corresponded with a four-month longer median
Figure 1. MeCP2 protein structure and Rett syndrome-causing mutations. (A) Human MeCP2 is expressed from either of two alternatively spliced transcripts that differ only in their inclusion of exon 2. Structures are traditionally referred to by their location within the sequence of MeCP2-e2, the slightly shorter protein isoform produced from translation initiating at exon 2, as shown (486 amino acids). The MBD was identified by deletion mapping to residues 78-162. The ability of MeCP2 to repress methylated reporters in vitro is dependent on amino acids 207-310 (TRD). The region between these domains is frequently referred to as the ID. (B) Conserved domains within MeCP2 are indicated on the basis of an alignment of human, mouse, rat, frog, and fish MECP2 orthologs. A large stretch of highly conserved amino acids overlaps with the MBD (amino acids 72-166). The two highly conserved AT-hooks are indicated, and a highly conserved stretch of histidines is located in the C-terminus. (C) Over 80 unique missense and 140 unique truncating mutations have been identified in girls with Rett syndrome. Recurrent, Rett-causing mutations are indicated along the MeCP2 protein sequence (150). Missense mutations are indicated above and are found within highly conserved motifs within the $\mathrm{MeCP} 2$ sequence. Truncating mutations are indicated below. Less common truncating mutations are not shown. Truncating mutations located before and including R270X are associated with more severe symptoms when compared with those of R294X or more C-terminal-truncating mutations, as indicated at the bottom of the diagram.

lifespan of G273X mice than that of R270X mice, consistent with patient outcomes (96). Interestingly, MeCP2 exhibits homology to the high-mobility group AT-hook (HMGA) family of chromatin modulators, which contain two or three AT-hooks connected by a flexible linker (96). The specialized ability of HMGA proteins to nonenzymatically induce DNA bending via their AT-hooks suggests that these motifs within $\mathrm{MeCP} 2$ may contribute to transcriptional function in addition to binding (Figure 2A and ref. 97).

$\mathrm{MeCP} 2$ in the chromatin context. MeCP2 is broadly expressed throughout the body but is most abundant in mature neurons. In fact, it is estimated that there are an average of 16 million MeCP2 molecules in a neuronal nucleus compared with 32 million nucleosomes (86). This astonishing abundance may be critical to understanding the role of $\mathrm{MeCP} 2$ in the nucleus and the singular failure of neurons upon its loss. By comparison, MeCP2 protein levels are approximately 30 times lower in liver nuclei and approximately seven times lower in glia (86). Additionally, MeCP2 protein levels are temporally regulated, such that they increase to a plateau during the time of neuronal maturation $(49,86,87)$. Given the near-nucleosome abundance of MeCP2 in neuronal nuclei and the ability of multiple regions of the protein to interact with DNA, it was not surprising that genome-wide ChIP analysis showed broad localization of MeCP2 throughout the genome (80, 86, 98, 99). Although MeCP2 enrichment tracks with DNA methylation ( $\mathrm{mCG}$ and $\mathrm{mCH}$ ), the enrichment of $\mathrm{MeCP} 2$ at the highly methylated major satellite repeats, for example, is only 2-3 times higher than that of the genomic average $(80,86)$. Although biochemically consistent, the broad chromatin localization of MeCP2 challenged many simple mechanistic models. Could a site-specific repressor be at near-nucleosome levels and broadly distributed across the genome? Recent work investigating the common R306C mutation (found in $\sim 5 \%$ of Rett syndrome patients), which falls at the edge of 


\section{A Altering chromatin structure}

Bending DNA with AT-hooks
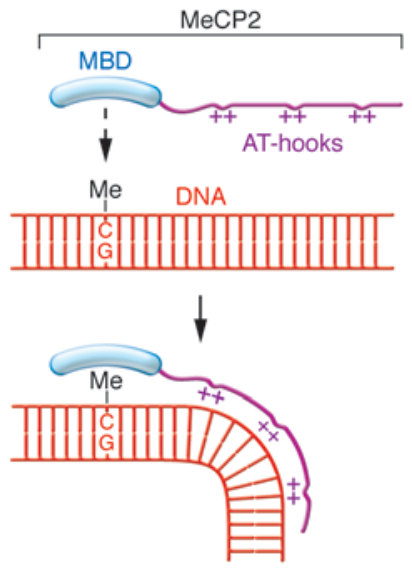

Antagonizing linker histone $\mathrm{H} 1$

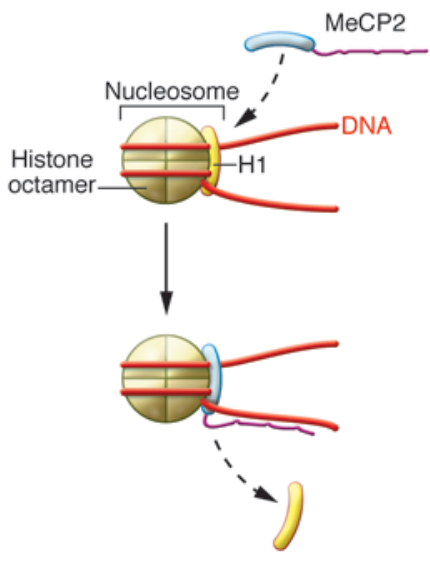

B Recruiting effector proteins

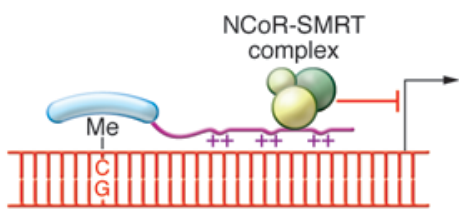

the TRD, found that this mutation ablates the interaction of MeCP2 with the nuclear receptor corepressor/SMRT (NCoR/SMRT) complex in vivo and in vitro (Figure 2B and refs. 100, 101). It remains to be seen whether NCoR/SMRT and MeCP2 colocalize at genes that are misregulated in mutants lacking either protein and what the functional overlap of misexpression is in the respective mutants. While this is an exciting molecular finding, patients with the R306C mutation have delayed onset of symptoms and decreased disease severity compared with patients who have MBD mutations or earlier truncations (Figure $1 \mathrm{C}$ and refs. 102-104). These findings are consistent with those in mouse models, suggesting that the NCoR interaction cannot fully explain the function of MeCP2.

The nuclear abundance and broad distribution of $\mathrm{MeCP} 2$ raise the possibility that it plays a structural role to affect chromatin. The fundamental structural unit of chromatin is the nucleosome, a histone octamer wrapped in 1.65 turns of DNA. In its most relaxed state, chromatin exists in a "beads-on-a-string" conformation, in which nucleosomes (beads) are separated by linker DNA (string). $\mathrm{MeCP} 2$ binds nucleosomes at the linker DNA entry/exit position (105), as does linker histone H1, which MeCP2 can displace (Figure $2 \mathrm{~A}$ and refs. 88,106$)$. Interestingly, the homology of MeCP2 to HMGA proteins is not limited to its AT-hook domain structure but extends to the ability of HMGA proteins to compete off H1 (107). As with the ability of histone $\mathrm{H} 1$ to promote higher-order chromatin structures $(108,109)$, purified MeCP2 is able to condense nucleosomal arrays at the apparently physiological ratio of $1 \mathrm{MeCP} 2$ to 2 nucleosomes (110). Increasing the amount of MeCP2 to 1 to 2 molecules per nucleosome produces even more compact ellipsoid structures, as determined by electron microscopy and sedimentation assays $(106,110)$. Antagonism between MeCP2 and H1 has been demonstrated in vivo, with KO neurons exhibiting a 2-fold
Figure 2. Functional modes of MeCP2. (A) One critical activity of MeCP2 is the binding of methylated cytosines through its MBD (blue). Once it binds DNA, MeCP2 may function by altering the chromatin structure. Left: AT-hooks are able to change the conformation of DNA. The presence of three AT-hooks in MeCP2 suggests that MeCP2 may be able to bend DNA like the HMGA proteins with which MeCP2 exhibits homology. In this scenario, the primary targeting of MeCP2 via the MBD would then allow the flexible C-terminus of the protein (purple) to engage proximal DNA via its positively charged AT-hooks. Right: The fundamental unit of chromatin is the nucleosome, a histone octamer wrapped in 1.65 turns of DNA (red). The DNA between nucleosomes is called linker DNA. Linker histone $\mathrm{H} 1$ (yellow) and MeCP2 share a common nucleosomal binding site, the linker DNA entry/exit position. MeCP2 is able to compete off histone $\mathrm{H} 1$ in vitro, like HMCA proteins, and neurons lacking MeCP2 display increased H1. H1 plays a critical role in establishing higher-order chromatin, suggesting that antagonism by MeCP2 affects chromatin architecture. (B) MeCP2 may alter transcription by recruiting effector proteins. MeCP2 is able to repress methylated reporters in vitro when its TRD is intact. Thus, the TRD (within the flexible, basic [-terminus [purple]) may function as a bridge between MBD (blue) targeting and transcriptional effectors. Recently, it was discovered that Rett patient mutation R306C (edge of the TRD) disrupts interaction of MeCP2 with the NCoR/SMRT corepressor complex.

increase in $\mathrm{H} 1$ (86). However, the structural ramifications of this antagonism and what transcriptional defects result from it are unknown. Higher-order chromatin structural defects have been observed in KO mice at a variety of candidate loci $(111,112)$, intimating the potential importance of a genome-wide assessment of chromatin interaction changes in KO mice (Figure 2).

With respect to changes in chromatin state, very few alterations have been reported by multiple independent groups, suggesting that they may be subtle in nature or context dependent. Uniquely, mislocalization of $\alpha$ thalassemia/mental retardation syndrome X-linked (ATRX) in KO neurons has been repeatedly noted $(96,101,112,113)$. It is interesting that this mislocalization has so far only been observed in the brain and that the timing of mislocalization tightly corresponds to disease progression in mouse models that develop symptoms at different rates (96, 101). Importantly, analysis of heterozygous-null females indicates that ATRX mislocalization is cell autonomous and, therefore, is unlikely to represent a general harbinger of circuit dysfunction (96). Loss-of-function mutations in ATRX result in severe intellectual disability (114) characterized by microcephaly, hypotonia, genital abnormalities, and seizures $(115,116)$. More work is necessary to determine whether ATRX mislocalization contributes to the pathology of Mecp2-mutant animals. However, hyperlocalization of ATRX in mice overexpressing MECP2 indicates that ATRX localization is a sensitive readout of MeCP2 function (96).

Finally, it is essential to note that neurons form a specialized class of postmitotic cells that constantly undergo bursts of activity in response to their environment. Although $\mathrm{MeCP} 2$ is subject to many activity-induced posttranslational modifications $(117,118)$, the functional role of MeCP2 upon neuronal activity is poorly understood in vivo. Knockin mice expressing a mutant form of MeCP2 
that cannot undergo activity-dependent phosphorylation at T308 exhibit microcephaly, a phenotype associated with hypomorphic alleles (118). Interestingly, cultured cortical neurons from these mice display decreased activity-induced expression, suggesting that MeCP2 may influence this process. Further, recent work using the odorant-evoked induction paradigm demonstrated defective transcriptional induction in olfactory sensory neurons of $\mathrm{KO}$ animals (119). However, much more research is needed to determine the molecular function of MeCP2 during the activity cycles of neurons and how a lack of this function might lead to disease.

\section{Therapeutic promise: getting back to the clinic}

Amelioration of the symptoms of sufferers of $M E C P 2$ disorders does not require absolute mechanistic knowledge of the molecular function of MeCP2. The reversibility of the phenotypes in the Rett mouse model established the potential benefit of therapeutic intervention in patients. Thus far, however, no interventions have fully rescued $\mathrm{KO}$ animals. While modest improvements have been observed, as described below, it is important to be cautiously optimistic while the transition is made to human patients, as the path to clinical translation is notoriously fraught with challenges. Fortunately, preclinical trials in patients with $M E C P 2$ disorders are strengthened by the face validity of both the Rett and MDS mouse models (see Tables 1 and 2) as well as by the continued focus within the community on best practices of blinding, randomization, and replication (120).

Initial attempts to rescue Rett mouse models focused on reversing the putative damage caused by the transcriptional changes observed in the KO animals. KO animals exhibit decreased RNA and protein levels of brain-derived neurotrophic factor (BDNF) $(89,121,122)$. Given the importance of BDNF as a neuronal growth factor, attempts have been made to normalize the levels of BDNF in KO animals genetically and pharmacologically. Overexpression of BDNF extends the median lifespan of KO animals by 3 weeks (121). Further, treatment with the ampakine CX546 increased BDNF in KO animals and restored respiratory frequency and respiratory minute volume to WT levels (123). Additionally, increasing BDNF in KO animals via administration of fingolimod resulted in total rescue of motor dysfunction, as measured by the ability to stay on the rotating rod, and a 3- to 4-week extension of median lifespan (124). Importantly, the BDNF V66M polymorphism in patients with Rett syndrome appears to modulate the severity of symptoms in humans (125). Thus, two small clinical trials are currently underway to explore the effects of FDA-approved copaxone (glatiramer acetate), which has been shown to increase the number of BDNF-expressing cells in KO animals (126), on outcomes in patients with Rett syndrome.

Given the low penetrance of BDNF across the blood-brain barrier, other studies have focused on similar growth factors that might be more clinically tractable. Specifically, administration of the tripeptide insulin-like growth factor 1 (IGF1) to KO animals resulted in an extension of lifespan similar to that observed with BDNF overexpression and partially rescues locomotor activity, breathing variability, spine density, and synaptic amplitude (127). Further, IGF1 treatment increased the number of glutamatergic synapses in neurons derived from induced pluripotent stem cells (iPSCs) from Rett syndrome patients (45). Although delivery of full-length human IGF1 (mecasermin) to KO animals resulted in a less robust phenotypic rescue than did the tripeptide formulation (128), a phase I clinical trial (NCT identifier 01253317) in Rett patients indicated good drug tolerance (129). The efficacy of this treatment will be clearer upon completion of the current phase II trials (NCT identifier 01777542).

Additional potential therapies have focused on neurotransmitter deficiencies. Given the decrease in dopamine metabolites in patients with Rett and in mouse models $(55,61,130)$ and the decreased dopaminergic activity in the substantia nigra observed in $\mathrm{KO}$ animals (56), the impact of levodopa (L-DOPA) therapy on KO animals was assessed. The locomotor activity of treated animals, as measured by distance traveled in the open-field assay, doubled (56). KO animals also exhibit decreased GABA levels in some areas of their brains $(57,131)$, and administration of the GABA reuptake inhibitor NO-711 resulted in a four-fold decrease in apnea frequency (132). Serotonin receptor agonists have also been successful in ameliorating the breathing abnormalities of $\mathrm{KO}$ animals $(132,133)$.

Strategies for the future. Future therapeutic strategies being explored for patients with Rett syndrome include gene therapy and reactivation of the $\mathrm{X}$ chromosome carrying the WT MECP2 allele. Neonatal bilateral intracranial injection of AAV9 containing MECP2 cDNA into $\mathrm{KO}$ animals resulted in $7 \%$ to $41 \%$ cellular transduction, depending on brain region, and extended median lifespan by 7 weeks compared with treatment with a virus encoding GFP (134). AAV9 was used, as it crosses the blood-brain barrier $(135,136)$; however, in the same study, tail-vein injection of the virus into 4 - to 5 -week old mice resulted in only $2 \%$ to $4 \%$ transduction. Strikingly, this minimal increase in MeCP2 levels appeared to extend the median lifespan by 5 weeks compared with that of uninjected animals (134). In a similar proof-of-concept study, AAV9 encoding Mecp2 was injected into the tails of 4to 6 -week-old KO animals, achieving transduction of $7 \%$ to $26 \%$ of cells in the brain regions assayed (137). Although each group only contained 5 animals, KO animals that received viral Mecp2 had a median lifespan that was approximately 15 weeks longer than that of mice injected with the viral control. Further, to better model gene therapy in patients with Rett syndrome, symptomatic heterozygous female mice were injected and showed continuous improvement in phenotypic severity scores, apnea frequency, and motor activity over a 20-week monitoring period (137). Of note, both of the above studies used viral DNA encoding the e1 isoform of $M E C P 2 / M e c p 2$, which does not include exon 2 and is more highly expressed in the brain than the 2 isoform containing exon 2 (138). Thus, while in its early stages of preclinical development, gene therapy may be an emergent therapeutic as long as the levels of MeCP2 delivered do not exceed the threshold for overexpression phenotypes. Additionally, although current analysis of patient mutations in mouse models (R111G, R270X, G273X, and R306C) indicates that these mutations are fully complemented by the WT allele, it is formally possible that some mutations in patients might be dominant-negative and require inhibition $(96,101)$.

One major concern with all therapies that target MeCP2 levels - whether delivering much-needed WT protein to Rett patients or decreasing the level of MeCP2 in sufferers of MDS - is maintaining the fine line of subclinical alterations in MeCP2 dosage. In a similar vein, increasing the dosage of more than a thousand $\mathrm{X}$-linked genes in females with Rett syndrome by reactivating 
an inactive $\mathrm{X}$ chromosome that may contain the repressed WT allele might seem dangerous, given the evolutionary drive to keep a single dose of most X-linked genes. However, recent work has uncovered a mouse mutation that results in biallelic expression of Mecp2, without increasing expression of the majority of X-linked genes (139). Thus, this work opens the door for possible selective dual expression of $M E C P 2$ in patients with Rett syndrome, ensuring that each neuron expresses the WT allele. While this work represents a large leap forward, further work is necessary to determine whether biallelic expression of even a few X-linked genes other than $M E C P 2$ is deleterious.

Although mouse models of MDS have not formally been tested for reversibility, the progressive nature of the disorder, the absence of neuronal death, and the reversibility of Mecp2 loss-offunction mutations suggest that this disorder might be ameliorated by decreasing MeCP2 levels. If MDS mouse models are rescued by halving the amount of $\mathrm{MeCP} 2$, many avenues of intervention might be fruitful, such as inhibition of proteins that stabilize or activate $\mathrm{MeCP}$, targeted destabilization of the $M E C P 2$ transcript, or inhibition of critical downstream pathways that are overactivated in MDS. Importantly, mice with a $50 \%$ decrease in MeCP2 levels exhibit measurable motor and behavioral dysfunction (140). Thus, treatments for MDS that decrease MeCP2 levels will require patient-specific dosing to ensure $\mathrm{MeCP} 2$ levels do not drop below the threshold that induces Rett-like symptoms.

\section{Outlook}

In the past 15 years, the development of mouse models of both Rett syndrome and MDS have enabled detailed behavioral, neu- rological, cellular, and molecular insights into these disorders that would not have otherwise been possible. Further, these robust models have revealed multiple potential therapeutic entry points, many of which are currently being explored in preclinical and clinical trials. Ongoing basic research into the molecular function of MeCP2 and the impact of its loss or gain will no doubt yield greater depth to our understanding of these disorders and unveil even more potential therapeutics. Thus, we are well into the promising stage of returning to the clinic with the knowledge we have gleaned at the bench.

\section{Acknowledgments}

The authors wish to dedicate this Review to the memory of Professor Bengt Hagberg (1923-2015), whose stellar clinical work inspired Huda Zoghbi to recognize the syndrome and pursue its etiology. We are very grateful to the families of individuals with Rett syndrome and MDS for their participation in our studies. Work by the authors has been supported by grants from the National Institute of Neurological Disorders and Stroke (NS057819), the Rett Syndrome Research Trust, and the International Rett Syndrome Foundation. L.M. Lombardi and H.Y. Zoghbi are also funded by the Howard Hughes Medical Institute. We apologize to the authors whose many fine studies on MeCP2 function we were unable to discuss due to space limitations and to the clinical focus of this work.

Address correspondence to: Huda Yahya Zoghbi, Baylor College of Medicine, One Baylor Plaza, Houston, Texas 77030, USA. Phone: 713.798.6523; E-mail: hzoghbi@bcm.edu.
1. Rett A. [On a unusual brain atrophy syndrome in hyperammonemia in childhood]. Wien Med Wochenschr. 1966;116(37):723-726.

2. Hagberg B, Aicardi J, Dias K, Ramos O. A progressive syndrome of autism, dementia, ataxia, and loss of purposeful hand use in girls: Rett's syndrome: report of 35 cases. Ann Neurol. 1983;14(4):471-479.

3. Trappe $\mathrm{R}$, et al. MECP2 mutations in sporadic cases of Rett syndrome are almost exclusively of paternal origin. Am J Hum Genet. 2001;68(5):1093-1101.

4. Amir RE, Van den Veyver IB, Wan M, Tran CQ, Francke U, Zoghbi HY. Rett syndrome is caused by mutations in X-linked MECP2, encoding methyl-CpG-binding protein 2. Nat Genet. 1999;23(2):185-188.

5. Van Esch H, et al. Duplication of the MECP2 region is a frequent cause of severe mental retardation and progressive neurological symptoms in males. Am J Hum Genet. 2005;77(3):442-453.

6. Hagberg B, Witt-Engerström I. Rett syndrome: a suggested staging system for describing impairment profile with increasing age towards adolescence. Am JMed Genet Suppl. 1986;1:47-59.

7. Hagberg B. Rett syndrome: long-term clinical follow-up experiences over four decades. JChild Neurol. 2005;20(9):722-727.

8. Roze E, et al. Rett syndrome: an overlooked diagnosis in women with stereotypic hand movements, psychomotor retardation, Parkinsonism, and dystonia? Mov Disord. 2007;22(3):387-389.

9. Armstrong DD. Neuropathology of Rett syndrome. J Child Neurol. 2005;20(9):747-753.

10. Salomao Schwartzman J, et al. Rett syndrome in a boy with a 47,XXY karyotype. Am J Hum Genet. 1999;64(6):1781-1785.

11. Tariverdian G, Kantner G, Vogel F. A monozygotic twin pair with Rett syndrome. Hum Genet. 1987;75(1):88-90.

12. Partington MW. Rett syndrome in monozygotic twins. Am J Med Genet. 1988;29(3):633-637.

13. Villard L, Kpebe A, Cardoso C, Chelly PJ, Tardieu PM, Fontes M. Two affected boys in a Rett syndrome family: clinical and molecular findings. Neurology. 2000;55(8):1188-1193.

14. Zoghbi HY, Percy AK, Schultz RJ, Fill C. Patterns of X chromosome inactivation in the Rett syndrome. Brain Dev. 1990;12(1):131-135.

15. Sirianni N, Naidu S, Pereira J, Pillotto RF, Hoffman EP. Rett syndrome: confirmation of X-linked dominant inheritance, and localization of the gene to Xq28. Am JHum Genet. 1998;63(5):1552-1558.

16. Chahrour M, Zoghbi HY. The story of Rett syndrome: from clinic to neurobiology. Neuron. 2007;56(3):422-437.

17. Neul JL, et al. Specific mutations in methyl-CpGbinding protein 2 confer different severity in Rett syndrome. Neurology. 2008;70(16):1313-1321.

18. Wan M, et al. Rett syndrome and beyond: recurrent spontaneous and familial MECP2 mutations at CpG hotspots. Am J Hum Genet. 1999;65(6):1520-1529.

19. Zeev BB, et al. Rett syndrome: clinical manifestations in males with MECP2 mutations. JChild Neurol. 2002;17(1):20-24.

20. Klauck SM, Lindsay S, Beyer KS, Splitt M, Burn J, Poustka A. A mutation hot spot for nonspecific $\mathrm{X}$-linked mental retardation in the MECP2 gene causes the PPM-X syndrome. Am J Hum Genet. 2002;70(4):1034-1037.

21. Cohen D, et al. MECP2 mutation in a boy with language disorder and schizophrenia. Am JPsychiatry. 2002;159(1):148-149.

22. Shibayama A, et al. MECP2 structural and 3'-UTR variants in schizophrenia, autism and other psychiatric diseases: a possible association with autism. Am JMed Genet B Neuropsychiatr Genet. 2004;128B(1):50-53.

23. Adegbola AA, Gonzales ML, Chess A, LaSalle JM, Cox GF. A novel hypomorphic MECP2 point mutation is associated with a neuropsychiatric phenotype. Hum Genet. 2009;124(6):615-623.

24. Venkateswaran S, McMillan HJ, Doja A, Humphreys P. Adolescent onset cognitive regression and neuropsychiatric symptoms associated with the A140V MECP2 mutation. Dev Med Child Neurol. 2014;56(1):91-94.

25. Carney RM, et al. Identification of MeCP2 mutations in a series of females with autistic disorder. Pediatr Neurol. 2003;28(3):205-211.

26. Campos M, et al. A MECP2 missense mutation 
within the MBD domain in a Brazilian male with autistic disorder. Brain Dev. 2011;33(10):807-809.

27. Li H, Yamagata T, Mori M, Yasuhara A, Momoi MY. Mutation analysis of methyl-CpG binding protein family genes in autistic patients. Brain Dev. 2005;27(5):321-325.

28. Wulffaert J, Van Berckelaer-Onnes IA, Scholte EM. Autistic disorder symptoms in Rett syndrome. Autism. 2009;13(6):567-581.

29. Gillberg C. Autistic symptoms in Rett syndrome: the first two years according to mother reports. Brain Dev. 1987;9(5):499-501.

30. Olsson B. Autistic traits in the Rett syndrome. Brain Dev. 1987;9(5):491-498.

31. Zappella M. Rett syndrome: a significant proportion of girls affected by autistic behavior. Brain Dev. 1985;7(3):307-312.

32. Neul JL. The relationship of Rett syndrome and MECP2 disorders to autism. Dialogues Clin Neurosci. 2012;14(3):253-262.

33. Meins $\mathrm{M}$, et al. Submicroscopic duplication in Xq28 causes increased expression of the MECP2 gene in a boy with severe mental retardation and features of Rett syndrome. JMed Genet. 2005;42(2):e12.

34. Bauters M, et al. Nonrecurrent MECP2 duplications mediated by genomic architecture-driven DNA breaks and break-induced replication repair. Genome Res. 2008;18(6):847-858.

35. Carvalho CM, et al. Complex rearrangements in patients with duplications of MECP2 can occur by fork stalling and template switching. Hum Mol Genet. 2009;18(12):2188-2203.

36. Lugtenberg D, et al. Structural variation in Xq28: MECP2 duplications in $1 \%$ of patients with unexplained XLMR and in $2 \%$ of male patients with severe encephalopathy. Eur J Hum Genet. 2009;17(4):444-453.

37. Friez MJ, et al. Recurrent infections, hypotonia, and mental retardation caused by duplication of MECP2 and adjacent region in Xq28. Pediatrics. 2006;118(6):e1687-e1695.

38. Ramocki MB, et al. Autism and other neuropsychiatric symptoms are prevalent in individuals with MeCP2 duplication syndrome. Ann Neurol. 2009;66(6):771-782.

39. Clayton-Smith J, et al. Xq28 duplication presenting with intestinal and bladder dysfunction and a distinctive facial appearance. Eur J Hum Genet. 2009;17(4):434-443.

40. Yang T, et al. Overexpression of methyl-CpG binding protein 2 impairs $\mathrm{T}(\mathrm{H}) 1$ responses. $\mathrm{Sci}$ Transl Med. 2012;4(163):163ra158.

41. Van Esch H. MECP2 duplication syndrome. Mol Syndromol. 2012;2(3):128-136.

42. Reardon W, et al. Progressive cerebellar degenerative changes in the severe mental retardation syndrome caused by duplication of MECP2 and adjacent loci on Xq28. Eur JPediatr. 2010;169(8):941-949.

43. Chen RZ, Akbarian S, Tudor M, Jaenisch R. Deficiency of methyl-CpG binding protein- 2 in CNS neurons results in a Rett-like phenotype in mice. Nat Genet. 2001;27(3):327-331.

44. Guy J, Hendrich B, Holmes M, Martin JE, Bird A. A mouse Mecp2-null mutation causes neurological symptoms that mimic Rett syndrome. Nat Genet. 2001;27(3):322-326.
45. Marchetto MC, et al. A model for neural development and treatment of Rett syndrome using human induced pluripotent stem cells. Cell. 2010;143(4):527-539.

46. Li Y, et al. Global transcriptional and translational repression in human-embryonic-stem-cellderived Rett syndrome neurons. Cell Stem Cell. 2013;13(4):446-458.

47. Armstrong D, Dunn JK, Antalffy B, Trivedi R. Selective dendritic alterations in the cortex of Rett syndrome. J Neuropathol Exp Neurol. 1995;54(2):195-201.

48. Fukuda T, Itoh M, Ichikawa T, Washiyama K, Goto Y. Delayed maturation of neuronal architecture and synaptogenesis in cerebral cortex of Mecp2-deficient mice. J Neuropathol Exp Neurol. 2005;64(6):537-544.

49. Kishi N, Macklis JD. MECP2 is progressively expressed in post-migratory neurons and is involved in neuronal maturation rather than cell fate decisions. Mol Cell Neurosci. 2004;27(3):306-321.

50. Schüle B, Armstrong DD, Vogel H, Oviedo A, Francke U. Severe congenital encephalopathy caused by MECP 2 null mutations in males: central hypoxia and reduced neuronal dendritic structure. Clin Genet. 2008;74(2):116-126.

51. Chapleau CA, et al. Dendritic spine pathologies in hippocampal pyramidal neurons from Rett syndrome brain and after expression of Rett-associated MECP2 mutations. Neurobiol Dis. 2009;35(2):219-233.

52. Asaka Y, Jugloff DG, Zhang L, Eubanks JH, Fitzsimonds RM. Hippocampal synaptic plasticity is impaired in the Mecp2-null mouse model of Rett syndrome. Neurobiol Dis. 2006;21(1):217-227.

53. Moretti $\mathrm{P}$, et al. Learning and memory and synaptic plasticity are impaired in a mouse model of Rett syndrome. J Neurosci. 2006;26(1):319-327.

54. Guy J, Gan J, Selfridge J, Cobb S, Bird A. Reversal of neurological defects in a mouse model of Rett syndrome. Science. 2007;315(5815):1143-1147.

55. Panayotis N, Ghata A, Villard L, Roux JC. Biogenic amines and their metabolites are differentially affected in the Mecp2-deficient mouse brain. BMC Neurosci. 2011;12:47.

56. Panayotis N, Pratte M, Borges-Correia A, Ghata A, Villard L, Roux JC. Morphological and functional alterations in the substantia nigra pars compacta of the Mecp2-null mouse. Neurobiol Dis. 2011;41(2):385-397.

57. El-Khoury R, Panayotis N, Matagne V, Ghata A, Villard L, Roux JC. GABA and glutamate pathways are spatially and developmentally affected in the brain of Mecp2-deficient mice. PLoS One. 2014;9(3):e92169.

58. Ide S, Itoh M, Goto Y. Defect in normal developmental increase of the brain biogenic amine concentrations in the mecp2-null mouse. Neurosci Lett. 2005;386(1):14-17.

59. Santos M, et al. Monoamine deficits in the brain of methyl-CpG binding protein 2 null mice suggest the involvement of the cerebral cortex in early stages of Rett syndrome. Neuroscience. 2010;170(2):453-467.

60. Zoghbi HY, et al. Cerebrospinal fluid biogenic amines and biopterin in Rett syndrome. Ann Neurol.1989;25(1):56-60.
61. Samaco RC, et al. Loss of MeCP2 in aminergic neurons causes cell-autonomous defects in neurotransmitter synthesis and specific behavioral abnormalities. Proc Natl Acad Sci U S A. 2009;106(51):21966-21971.

62. Ramaekers VT, et al. Reduced folate transport to the CNS in female Rett patients. Neurology. 2003;61(4):506-515.

63. Fyffe SL, et al. Deletion of Mecp2 in Sim1expressing neurons reveals a critical role for $\mathrm{MeCP} 2$ in feeding behavior, aggression, and the response to stress. Neuron. 2008;59(6):947-958.

64. Chao HT, et al. Dysfunction in GABA signalling mediates autism-like stereotypies and Rett syndrome phenotypes. Nature. 2010;468(7321):263-269.

65. He LJ, et al. Conditional deletion of Mecp2 in parvalbumin-expressing GABAergic cells results in the absence of critical period plasticity. Nat Commun. 2014;5:5036.

66. Lioy DT, et al. A role for glia in the progression of Rett's syndrome. Nature. 2011;475(7357):497-500.

67. Cronk JC, et al. Methyl-CpG binding protein 2 regulates microglia and macrophage gene expression in response to inflammatory stimuli. Immunity. 2015;42(4):679-691.

68. Luikenhuis S, Giacometti E, Beard CF, Jaenisch R. Expression of $\mathrm{MeCP} 2$ in postmitotic neurons rescues Rett syndrome in mice. Proc Natl Acad Sci US A. 2004;101(16):6033-6038.

69. Giacometti E, Luikenhuis S, Beard C, Jaenisch R. Partial rescue of MeCP2 deficiency by postnatal activation of MeCP2. Proc Natl Acad Sci U S A. 2007;104(6):1931-1936.

70. McGraw CM, Samaco RC, Zoghbi HY. Adult neural function requires MeCP2. Science. 2011;333(6039):186.

71. Robinson L, et al. Morphological and functional reversal of phenotypes in a mouse model of Rett syndrome. Brain. 2012;135(pt 9):2699-2710.

72. Collins AL, et al. Mild overexpression of MeCP2 causes a progressive neurological disorder in mice. Hum Mol Genet. 2004;13(21):2679-2689.

73. Samaco RC, Mandel-Brehm C, McGraw CM, Shaw CA, McGill BE, Zoghbi HY. Crh and Oprm1 mediate anxiety-related behavior and social approach in a mouse model of MECP2 duplication syndrome. Nat Genet. 2012;44(2):206-211.

74. del Gaudio D, et al. Increased MECP2 gene copy number as the result of genomic duplication in neurodevelopmentally delayed males. Genet Med. 2006;8(12):784-792.

75. Chao HT, Zoghbi HY, Rosenmund C. MeCP2 controls excitatory synaptic strength by regulating glutamatergic synapse number. Neuron. 2007;56(1):58-65.

76. Lewis JD, et al. Purification, sequence, and cellular localization of a novel chromosomal protein that binds to methylated DNA. Cell. 1992;69(6):905-914.

77. Nan X, Meehan RR, Bird A. Dissection of the methyl-CpG binding domain from the chromosomal protein MeCP2. Nucleic Acids Res. 1993;21(21):4886-4892.

78. Guo JU, et al. Distribution, recognition and regulation of non-CpG methylation in the adult mammalian brain. Nat Neurosci. 2014;17(2):215-222 .

79. Gabel HW, et al. Disruption of DNA-methyla- 
tion-dependent long gene repression in Rett syndrome. Nature. 2015;522(7554):89-93.

80. Chen L, et al. MeCP2 binds to non-CG methylated DNA as neurons mature, influencing transcription and the timing of onset for Rett syndrome. Proc Natl Acad Sci U S A. 2015;112(17):5509-5514.

81. Smith ZD, Meissner A. DNA methylation: roles in mammalian development. Nat Rev Genet. 2013;14(3):204-220.

82. Schubeler D. Function and information content of DNA methylation. Nature. 2015;517(7534):321-326.

83. Jones PA. Functions of DNA methylation: islands, start sites, gene bodies and beyond. Nat Rev Genet. 2012;13(7):484-492.

84. Baubec T, et al. Genomic profiling of DNA methyltransferases reveals a role for DNMT3B in genic methylation. Nature. 2015;520(7546):243-247.

85. Lister R, et al. Global epigenomic reconfiguration during mammalian brain development. Science. 2013;341(6146):1237905.

86. Skene PJ, et al. Neuronal MeCP2 is expressed at near histone-octamer levels and globally alters the chromatin state. Mol Cell. 2010;37(4):457-468.

87. Shahbazian MD, Antalffy B, Armstrong DL, Zoghbi HY. Insight into Rett syndrome: MeCP2 levels display tissue- and cell-specific differences and correlate with neuronal maturation. Hum Mol Genet. 2002;11(2):115-124.

88. Nan X, Campoy FJ, Bird A. MeCP2 is a transcriptional repressor with abundant binding sites in genomic chromatin. Cell. 1997;88(4):471-481.

89. Chahrour M, et al. MeCP2, a key contributor to neurological disease, activates and represses transcription. Science. 2008;320(5880):1224-1229.

90. Ben-Shachar S, Chahrour M, Thaller C, Shaw CA, Zoghbi HY. Mouse models of MeCP2 disorders share gene expression changes in the cerebellum and hypothalamus. Hum Mol Genet. 2009;18(13):2431-2442.

91. Yazdani M, Deogracias R, Guy J, Poot RA, Bird A, Barde YA. Disease modeling using embryonic stem cells: $\mathrm{MeCP} 2$ regulates nuclear size and RNA synthesis in neurons. Stem Cells. 2012;30(10):2128-2139.

92. Adams VH, McBryant SJ, Wade PA, Woodcock CL, Hansen JC. Intrinsic disorder and autonomous domain function in the multifunctional nuclear protein, MeCP2.J Biol Chem. 2007;282(20):15057-15064.

93. Wakefield RI, et al. The solution structure of the domain from $\mathrm{MeCP} 2$ that binds to methylated DNA. J Mol Biol. 1999;291(5):1055-1065.

94. Ho KL, McNae IW, Schmiedeberg L, Klose RJ, Bird AP, Walkinshaw MD. MeCP2 binding to DNA depends upon hydration at methyl-CpG. Mol Cell. 2008;29(4):525-531.

95. Ghosh RP, et al. Unique physical properties and interactions of the domains of methylated DNA binding protein 2. Biochemistry. 2010;49(20):4395-4410.

96. Baker SA, Chen L, Wilkins AD, Yu P, Lichtarge O, Zoghbi HY. An AT-hook domain in MeCP2 determines the clinical course of Rett syndrome and related disorders. Cell. 2013;152(5):984-996.

97. Reeves R, Beckerbauer L. HMGI/Y proteins: flexible regulators of transcription and chromatin structure. Biochim Biophys Acta. 2001;1519(1):13-29.
98. Yasui DH, et al. Integrated epigenomic analyses of neuronal MeCP2 reveal a role for long-range interaction with active genes. Proc Natl Acad Sci US A. 2007;104(49):19416-19421.

99. Cohen S, et al. Genome-wide activity-dependent $\mathrm{MeCP} 2$ phosphorylation regulates nervous system development and function. Neuron. 2011;72(1):72-85.

100.Lyst MJ, et al. Rett syndrome mutations abolish the interaction of MeCP2 with the NCoR/SMRT co-repressor. Nat Neurosci. 2013;16(7):898-902.

101. Heckman LD, Chahrour MH, Zoghbi HY. Rettcausing mutations reveal two domains critical for $\mathrm{MeCP} 2$ function and for toxicity in MECP2 duplication syndrome mice. Elife. 2014;3:e02676.

102.Schanen C, et al. Phenotypic manifestations of MECP2 mutations in classical and atypical Rett syndrome. Am JMed Genet A. 2004;126A(2):129-140.

103. Robertson L, Hall SE, Jacoby P, Ellaway C, de Klerk N, Leonard $\mathrm{H}$. The association between behavior and genotype in Rett syndrome using the Australian Rett Syndrome Database. Am JMed Genet B Neuropsychiatr Genet. 2006;141B(2):177-183.

104.Neul JL, et al. Developmental delay in Rett syndrome: data from the natural history study. J Neurodev Disord. 2014;6(1):20.

105. Nikitina T, Ghosh RP, Horowitz-Scherer RA, Hansen JC, Grigoryev SA, Woodcock CL. $\mathrm{MeCP} 2$-chromatin interactions include the formation of chromatosome-like structures and are altered in mutations causing Rett syndrome. J Biol Chem. 2007;282(38):28237-28245.

106. Ghosh RP, Horowitz-Scherer RA, Nikitina T, Shlyakhtenko LS, Woodcock CL. MeCP2 binds cooperatively to its substrate and competes with histone $\mathrm{H} 1$ for chromatin binding sites. Mol Cell Biol. 2010;30(19):4656-4670.

107. Zhao K, Kas E, Gonzalez E, Laemmli UK. SAR-dependent mobilization of histone H1 by HMG-I/Y in vitro: HMG-I/Y is enriched in H1-depleted chromatin. EMBO J. 1993;12(8):3237-3247.

108. Bednar J, et al. Nucleosomes, linker DNA, and linker histone form a unique structural motif that directs the higher-order folding and compaction of chromatin. Proc Natl Acad Sci U S A. 1998;95(24):14173-14178.

109. Harshman SW, Young NL, Parthun MR, Freitas MA. H1 histones: current perspectives and challenges. Nucleic Acids Res. 2013;41(21):9593-9609.

110. Georgel PT, Horowitz-Scherer RA, Adkins N, Woodcock CL, Wade PA, Hansen JC. Chromatin compaction by human MeCP2. Assembly of novel secondary chromatin structures in the absence of DNA methylation. J Biol Chem. 2003;278(34):32181-32188.

111. Horike S, Cai S, Miyano M, Cheng JF, KohwiShigematsu T. Loss of silent-chromatin looping and impaired imprinting of DLX5 in Rett syndrome. Nat Genet. 2005;37(1):31-40.

112. Kernohan KD, Vernimmen D, Gloor GB, Berube NG. Analysis of neonatal brain lacking ATRX or $\mathrm{MeCP} 2$ reveals changes in nucleosome density, CTCF binding and chromatin looping. Nucleic Acids Res. 2014;42(13):8356-8368.

113. Nan $X$, et al. Interaction between chromatin pro- teins MECP2 and ATRX is disrupted by mutations that cause inherited mental retardation. Proc Natl Acad Sci U S A. 2007;104(8):2709-2714.

114. Gibbons RJ, Picketts DJ, Villard L, Higgs DR. Mutations in a putative global transcriptional regulator cause $\mathrm{X}$-linked mental retardation with $\alpha$-thalassemia (ATR-X syndrome). Cell. 1995;80(6):837-845.

115. Gibbons R. $\alpha$-Thalassaemia-mental retardation, X linked. Orphanet J Rare Dis. 2006;1:15.

116. De La Fuente R, Baumann C, Viveiros MM. Role of ATRX in chromatin structure and function: implications for chromosome instability and human disease. Reproduction. 2011;142(2):221-234.

117. Zhou Z, et al. Brain-specific phosphorylation of $\mathrm{MeCP} 2$ regulates activity-dependent Bdnf transcription, dendritic growth, and spine maturation. Neuron. 2006;52(2):255-269.

118. Ebert DH, et al. Activity-dependent phosphorylation of MeCP2 threonine 308 regulates interaction with NCoR. Nature. 2013;499(7458):341-345.

119. Lee W, et al. MeCP2 regulates activity-dependent transcriptional responses in olfactory sensory neurons. Hum Mol Genet. 2014;23(23):6366-6374.

120. Katz DM, et al. Preclinical research in Rett syndrome: setting the foundation for translational success. Dis Model Mech. 2012;5(6):733-745.

121. Chang Q, Khare G, Dani V, Nelson S, Jaenisch R. The disease progression of Mecp2 mutant mice is affected by the level of BDNF expression. Neuron. 2006;49(3):341-348.

122. Wang $\mathrm{H}$, et al. Dysregulation of brain-derived neurotrophic factor expression and neurosecretory function in Mecp2 null mice. J Neurosci. 2006;26(42):10911-10915.

123. Ogier M, Wang H, Hong E, Wang Q, Greenberg ME, Katz DM. Brain-derived neurotrophic factor expression and respiratory function improve after ampakine treatment in a mouse model of Rett syndrome. J Neurosci. 2007;27(40):10912-10917.

124. Deogracias R, et al. Fingolimod, a sphingosine-1 phosphate receptor modulator, increases BDNF levels and improves symptoms of a mouse model of Rett syndrome. Proc Natl Acad Sci U S A. 2012;109(35):14230-14235.

125. Zeev BB, et al. The common BDNF polymorphism may be a modifier of disease severity in Rett syndrome. Neurology. 2009;72(14):1242-1247.

126. Ben-Zeev B, Aharoni R, Nissenkorn A, Arnon R. Glatiramer acetate (GA, Copolymer-1) an hypothetical treatment option for Rett syndrome. Med Hypotheses. 2011;76(2):190-193.

127. Tropea D, et al. Partial reversal of Rett Syndromelike symptoms in MeCP2 mutant mice. Proc Natl Acad Sci U S A. 2009;106(6):2029-2034.

128. Castro J, et al. Functional recovery with recombinant human IGF1 treatment in a mouse model of Rett Syndrome. Proc Natl Acad Sci U S A 2014;111(27):9941-9946.

129. Khwaja OS, et al. Safety, pharmacokinetics, and preliminary assessment of efficacy of mecasermin (recombinant human IGF-1) for the treatment of Rett syndrome. Proc Natl Acad Sci U S A. 2014;111(12):4596-4601.

130.Zoghbi HY, Percy AK, Glaze DG, Butler IJ, Riccardi VM. Reduction of biogenic amine levels in the Rett syndrome. $N$ Engl J Med. 1985;313(15):921-924. 
131. Medrihan L, et al. Early defects of GABAergic synapses in the brain stem of a MeCP2 mouse model of Rett syndrome. J Neurophysiol. 2008;99(1):112-121.

132. Abdala AP, Dutschmann M, Bissonnette JM, Paton JF. Correction of respiratory disorders in a mouse model of Rett syndrome. Proc Natl Acad Sci U S A. 2010;107(42):18208-18213.

133. Levitt ES, Hunnicutt BJ, Knopp SJ, Williams JT, Bissonnette JM. A selective 5-HT1a receptor agonist improves respiration in a mouse model of Rett syndrome. J Appl Physiol. 2013;115(11):1626-1633.

134. Gadalla KK, et al. Improved survival and reduced phenotypic severity following AAV9/MECP2 gene transfer to neonatal and juvenile male Mecp2 knockout mice. Mol Ther. 2013;21(1):18-30.

135. Gray SJ, Matagne V, Bachaboina L, Yadav S, Ojeda SR, Samulski RJ. Preclinical differences of intravascular AAV9 delivery to neurons and glia: a comparative study of adult mice and nonhuman primates. Mol Ther. 2011;19(6):1058-1069.

136. Foust KD, Nurre E, Montgomery CL, Hernandez A, Chan CM, Kaspar BK. Intravascular AAV9 preferentially targets neonatal neurons and adult astrocytes. Nat Biotechnol. 2009;27(1):59-65.

137. Garg SK, et al. Systemic delivery of MeCP2 rescues behavioral and cellular deficits in female mouse models of Rett syndrome. J Neurosci.
2013;33(34):13612-13620.

138. Dragich JM, Kim YH, Arnold AP, Schanen NC. Differential distribution of the MeCP2 splice variants in the postnatal mouse brain. JComp Neurol. 2007;501(4):526-542.

139. Bhatnagar S, et al. Genetic and pharmacological reactivation of the mammalian inactive X chromosome. Proc Natl Acad Sci US A. 2014;111(35):12591-12598.

140.Samaco RC, et al. A partial loss of function allele of methyl-CpG-binding protein 2 predicts a human neurodevelopmental syndrome. Hum Mol Genet. 2008;17(12):1718-1727.

141. Stearns NA, Schaevitz LR, Bowling H, Nag N, Berger UV, Berger-Sweeney J. Behavioral and anatomical abnormalities in Mecp2 mutant mice: a model for Rett syndrome. Neuroscience. 2007;146(3):907-921.

142. Samaco RC, McGraw CM, Ward CS, Sun Y, Neul JL, Zoghbi HY. Female Mecp2(+/-) mice display robust behavioral deficits on two different genetic backgrounds providing a framework for preclinical studies. Hum Mol Genet. 2013;22(1):96-109.

143. Shahbazian M, et al. Mice with truncated MeCP2 recapitulate many Rett syndrome features and display hyperacetylation of histone H3. Neuron. 2002;35(2):243-254.

144.Pelka GJ, et al. Mecp2 deficiency is associated with learning and cognitive deficits and altered gene activity in the hippocampal region of mice. Brain. 2006;129(pt 4):887-898.

145. Taneja P, Ogier M, Brooks-Harris G, Schmid DA, Katz DM, Nelson SB. Pathophysiology of locus ceruleus neurons in a mouse model of Rett syndrome. J Neurosci. 2009;29(39):12187-12195.

146.Voituron N, Zanella S, Menuet C, Dutschmann M, Hilaire G. Early breathing defects after moderate hypoxia or hypercapnia in a mouse model of Rett syndrome. Respir Physiol Neurobiol. 2009;168(1):109-118.

147. Bissonnette JM, Knopp SJ. Effect of inspired oxygen on periodic breathing in methy-CpG-binding protein 2 (Mecp2) deficient mice. JAppl Physiol. 2008;104(1):198-204.

148. Lang M, et al. Rescue of behavioral and EEG deficits in male and female Mecp2-deficient mice by delayed Mecp2 gene reactivation. Hum Mol Genet. 2014;23(2):303-318.

149.D'Cruz JA, Wu C, Zahid T, El-Hayek Y, Zhang L, Eubanks JH. Alterations of cortical and hippocampal EEG activity in MeCP2-deficient mice. Neurobiol Dis. 2010;38(1):8-16.

150. Christodoulou J, Grimm A, Maher T, Bennetts B. RettBASE: The IRSA MECP2 variation database-a new mutation database in evolution. Hum Mutat. 2003;21(5):466-472. 